COPYRIGHT @ 2018 INTERNATIONAL JOURNAL OF SCIENCE DENTISTRY | AVAILABLE ONLINE

http://www.periodicos.uff.br/index

\title{
CONSUMO DE ÁlCOOL PELO PACIENTE ODONTOLÓGICO: COMO E POR QUE QUANTIFICAR?
}

\section{ALCOHOL CONSUMPTION BY DENTAL PATIENT: HOW AND WHY \\ QUANTIFYING?}

William Simões de Oliveira

Especialista em Implantodontia - Hospital de Aeronáutica dos Afonsos - HAAF - Rio de Janeiro, RJ, Brasil.

Telma Regina Aguiar

Professora Adjunta - PhD em Periodontia - Faculdade de Odontologia - Universidade Federal Fluminense, Niterói, RJ, Brasil.

Priscila Ladeira Casado

Professora Adjunta - PhD em Morfologia - Faculdade de Odontologia - Universidade Federal Fluminense, Niterói, RJ, Brasil.

Endereço para Correspondência:

Rua Mario Santos Braga, 28 Centro, Niterói

Campus do Valonguinho

CEP :24020-140 E-mail: plcasado@hotmail.com

Categoria: Revisão de Literatura

Palavras chave: alcoolismo, consumo de álcool, paciente, odontológico, questionário

Keywords: alcohol consumption , patient, dentistry , questionnaire 
COPYRIGHT @ 2018 INTERNATIONAL JOURNAL OF SCIENCE DENTISTRY | AVAILABLE ONLINE http://www.periodicos.uff.br/index

\section{Introdução}

O álcool é uma droga lícita e socialmente aceita, mas seu uso pode causar prejuízos físicos, psíquicos e sociais, tanto no consumo crônico quanto no agudo. Há uma grande variedade de bebidas alcoólicas espalhadas pelo mundo, fazendo do álcool a substância psicoativa mais popular do planeta. Obtido por fermentação ou destilação da glicose presente em cereais, raízes e frutas, o etanol (ou álcool etílico) é consumido exclusivamente por via oral. O Brasil detém o primeiro lugar do mundo no consumo de destilados de cachaça e é o quinto maior produtor de cerveja da qual, só a Ambev, produz 35 milhões de garrafas por dia (ROCHA, 2011).

$\mathrm{Na}$ odontologia, sabe-se que o consumo de álcool é um dos principais fatores derisco relacionados ao desenvolvimento do câncer deboca. O álcool é a droga que mais agride o organismo como um todo (tanto quanto a cocaína e o craque); é a que mais faz vítimas; sendo a mais consumida entre os jovens no Brasil. O índice de câncer entre os bebedores é alarmante.O dano provocado pelo consumo de álcool namucosa oral pode ser resultado de sua ação direta, pelasua presença na corrente sanguínea ou de sua atuaçãosobre outros sistemas (ROCHA, 2011).Porém, o cirurgião-dentista pouco infere sobre os hábitos de consumo de álcool do paciente odontológico, e quando presente na anamnese é feito de maneira superficial, sem parâmetros para quantificar o consumo.Sendo assim, o objetivo deste trabalho é apresentar 6 questionários práticos, mais utilizados mundialmente, que foram desenvolvidos para ajudar a levantar a suspeita de problemas com o álcool. Estarevisão de literaturarelacionará as principais vantagens e desvantagens de cada questionário em relação à quantificação do consumo de álcool pelos pacientes.

\section{Revisão da Literatura}

Segundo Rosário(2011), na escolha de instrumentos de avaliação de consumo de álcool deve-se considerar o tempo a ser investido para as entrevistas e avaliação dos resultados.

1. Questionário AUDIT

O AUDIT é composto por 10 questões e foi desenvolvido pela

OMS(Organização Mundial de Saúde) baseando-se em estudos conduzidos na Austrália, Quênia, Noruega, Bulgária, México e Estados Unidos (BABOR et al., 1992; 
COPYRIGHT @ 2018 INTERNATIONAL JOURNAL OF SCIENCE DENTISTRY | AVAILABLE ONLINE http://www.periodicos.uff.br/index

SAUNDERS et al., 1993b). A versão em português brasileira do AUDIT foi proposta por MENDÉZ (1999) e, posteriormente, validada por Limaet al. (2005).

O AUDIT é um instrumento que avalia problemas associados ao consumo de álcool e detecta o padrão de consumo de risco. É, particularmente, indicado para atenção primária à saúde e utilizado por diferentes países, em diversos grupos culturais. O ponto de corte para determinação de consumo de beber com risco é 8 ou mais pontos. Este padrão de consumo de risco está associado às consequiências médicas, problemas psicológicos e sociais(DAWE et al., 2002; KNIBBE et al., 2006; CROTON, 2007). Este instrumento é capaz de identificar a quantidade, freqüência de consumo de bebida e dependência. As três primeiras questões permitem estimar o uso de álcool e as demais mensuram as conseqüências relacionadas ao consumo (DAWE et al., 2002; KNIBBE et al., 2006). O AUDIT diferencia-se de outros instrumentos de triagem para alcoolismo, pois enfatiza a identificação do consumo de risco em lugar da dependência de longa duração, podendo autoaplicado ou respondido por entrevista. Seu preenchimento pode ser concluído de 2-4 minutos. As pontuações do AUDIT se correlacionam positivamente com as medidas de consequiências do consumo, alterações de comportamento, vulnerabilidade à dependência de álcool e estados de ânimo negativos (BABOR et al., 1992; CROTON, 2007) (Tabela 1). O escore obtido no questionário AUDIT classifica o paciente em: baixo risco ou abstêmio (até 7 pontos), usuário de risco (de 8 a 15 pontos), uso nocivo (de 16 a 19 pontos) e dependência química (acima de 20 pontos). 
Tabela 1. Questionário AUDIT.

1. Com que freqüência (quantas vezes por semana) você consome bebidas alcoólicas?

( ) Nunca [0]( ) Uma vez por mês ou menos[1]( ) 2-4 vezes por mês[2]( ) 2-3 vezes por semana[3]

( ) 4 ou mais vezespor semana[4]

2. Quantas doses de álcool você consome num dia normal?

( ) 0 ou 1 [0]( ) 2 ou 3 [1]( ) 4 ou 5 [2]( ) 6 ou 7 [3]( ) 8 ou mais [4]

3. Com que freqüência (quantas vezes por semana) você consome cinco ou mais doses em uma única ocasião?

( ) Nunca [0]( ) Menos que uma vez por mês [1]( ) Uma vez por mês[2]( ) Uma vez por semana [3]

( ) Quase todos os dias [4]

4. Quantas vezes ao longo dos últimos doze meses você achou que não conseguiria parar de beber, uma vez tendo começado?

( ) Nunca [0]( ) Menos que uma vez por mês [1]( ) Uma vez por mês[2]( ) Uma vez por semana [3]

( ) Quase todos os dias [4]

5. Quantas vezes ao longo dos últimos doze meses você não conseguiu fazer o que era esperado de você por causa do álcool?

( ) Nunca [0]( ) Menos que uma vez por mês [1]( ) Uma vez por mês[2]( ) Uma vez por semana [3]

( ) Quase todos os dias [4]

6. Quantas vezes ao longo dos últimos doze meses você precisou beber pela manhã para poder se sentir bem ao longo do dia, após ter bebido bastante no dia anterior?

( ) Nunca [0]( ) Menos que uma vez por mês [1]( ) Uma vez por mês[2]( ) Uma vez por semana [3]

( ) Quase todos os dias [4]

7. Quantas vezes ao longo dos últimos doze meses você se sentiu culpado ou com remorso após ter bebido?

( ) Nunca [0]( ) Menos que uma vez por mês [1]( ) Uma vez por mês[2]( ) Uma vez por semana [3]

( ) Quase todos os dias [4]

8. Quantas vezes ao longo dos últimos doze meses você foi incapaz de lembrar o que aconteceu devido à bebida?

( ) Nunca [0]( ) Menos que uma vez por mês [1]( ) Uma vez por mês[2]( ) Uma vez por semana [3]

( ) Quase todos os dias [4]

9. Você já causou ferimentos ou prejuízos a você mesmo ou a outra pessoa após ter bebido?
( ) Não [0]
( ) Sim, mas não no último ano[2]( ) Sim, durante o último ano [4]

10. Alguém ou algum parente, amigo ou médico, já se preocupou com o fato de você beber ou sugeriu que você parasse?

( ) Não [0]( ) Sim, mas não no último ano[2]( ) Sim, durante o último ano [4]

\section{Questionário CAGE}

O CAGE (questionário de rastreamento de dependência de álcool, bastante disseminado, cuja sigla é o acrônimo das palavras inglesas Cutdown, Annoyed, Guilte Eye-opener)é composto por quatro questões, um instrumento simples e breve (menos de 1 minuto). A partir de uma resposta afirmativa suspeita-se de abuso de álcool, duas respostas afirmativas há indícios de dependência de álcool (DAWE et al., 2002; CROTON, 2007). Foi proposto por Mayfieldet al. (1974) na língua inglesa e traduzido para o português por Masur e Monteiro (1983). O CAGE é menos sensível para determinar consumo abusivo de álcool entre os mais jovens e em populações, e os casos de abuso de álcool nos últimos 12 meses ou mais recentes. Quando acompanhado de questões sobre quantidade e freqüência de consumo de álcool, torna-se um instrumento útil para ser incorporado às rotinas médicas ou entrevistas psicológicas (DAWE et al., 2002; PILLON SCet al., 2003; CROTON, 2007) (Tabela 2). 
COPYRIGHT @ 2018 INTERNATIONAL JOURNAL OF SCIENCE DENTISTRY | AVAILABLE ONLINE

http://www.periodicos.uff.br/index

Tabela 2. Questionário CAGE.

1. Você já tentou diminuir ou cortar ("Cutdown") a bebida?

2. Você já ficou incomodado ou irritado ("Annoyed") com outros porque criticaram seu jeito de beber?

3. Você já se sentiu culpado ("Guilty") por causa do seu jeito de beber?

4. Você já teve que beber para aliviar os nervos ou reduzir os efeitos de uma ressaca ("Eye-opener")

\section{Questionário Brief-MAST}

O questionário Brief-MAST (Teste de Detecção de Alcoolismo de Michigan, versão breve) desenvolvido por Pokornyet al.(1972) consiste de 10 perguntas, com respostas "sim" ou "não", que recebem pontuação e classificação de acordo com o seguinte escore: soma dos pontos $\leq 3$ significa ausência de problema com bebidas alcoólicas, $=4$ sugere alcoolismo e $\geq 5$ indica alcoolismo (Tabela 3 ).

Tabela 3. Questionário Brief-MAST(Teste de Detecção de Alcoolismo de Michigan, versão Breve).

1. Você se considera uma pessoa que bebe de modo normal? (Sim=0, Não=2)

2. Seus amigos ou parentes acham que você bebe de modo normal? ( $\mathrm{Sim}=0$, Não=2)

3. Você já foi a algum encontro dos Alcoólicos Anônimos (AA)? ( $\mathrm{Sim}=5$, Não=0)

4. Você já perdeu amigos/amigas ou namorado/namorada por causa da bebida? ( $\mathrm{Sim}=2$, Não=0)

5. Você já teve problemas no trabalho/emprego por causa da bebida? ( $\mathrm{Sim}=2, \mathrm{Não}=0)$

6. Você já abandonou suas obrigações, sua família ou seu trabalho por 2 ou mais dias em seguida por causa da bebida? $(\mathrm{Sim}=2, \mathrm{Não}=0)$

7. Você já teve delirium tremens, tremores, ouviu vozes, viu coisas que não estavam lá depois de beber muito? $(\operatorname{Sim}=2, \mathrm{Não}=0)$

8. Você já procurou algum tipo de ajuda por causa da bebida? $(\mathrm{Sim}=5$, Não=0)

9. Você já foi hospitalizado por causa da bebida? ( $\mathrm{Sim}=5$, Não=0)

10. Você já esteve preso ou foi multado por dirigir embriagado? ( $\mathrm{Sim}=2$, Não=0)

\section{Questionário T-ACE}

Sokolet al (1989) desenvolveram o T-ACE (acrônimo obtido das palavras inglesas: Tolerance, Annoyed, Cutdown e Eye-opener), um questionário breve semelhante ao CAGE. O T-ACE foi traduzido para o português a partir do texto original de Sokolet al (1989). Aplicável em um a dois minutos de conversação, o T-ACE além de viabilizar a detecção de gestantes que possuem consumo alcoólico de risco, mostrouse mais eficiente, com maior especificidade (89\%) e sensibilidade (69\%) que o CAGE e o MAST (Michigan AlcoholScreening Test). O Escore de 2 pontos ou mais classifica o paciente como de alta propensão ao consumo alcoólico de risco (Tabela 4). 
COPYRIGHT @ 2018 INTERNATIONAL JOURNAL OF SCIENCE DENTISTRY | AVAILABLE ONLINE

http://www.periodicos.uff.br/index

Tabela 4. Questionário T-ACE.

T- Qual a quantidade que você precisa beber para se sentir desinibido(a) ou mais alegre?

$$
\text { Não Bebo - } 0 \text { /Até duas doses }-1 \text { ponto / Três ou mais doses }-2 \text { pontos }
$$

A -Alguém tem lhe incomodado por criticar seu modo de beber?

$$
\text { Não }-0 / \operatorname{Sim}-1 \text { ponto }
$$

C- Você tem percebido que deve diminuir o seu consumo de bebidas?

$$
\text { Não - 0/ } \operatorname{Sim}-1 \text { ponto }
$$

E- Você costuma tomar bebida alcoólica logo pela manhã para manter-se bem ou para livrar-se do mal estar do dia anterior?

$$
\text { Não }-0 / \operatorname{Sim}-1 \text { ponto }
$$

\section{Questionário TWEAK}

O questionário TWEAKfoi usado para triagem de consumo alcoólico de risco entre pacientes ambulatoriais gestantes (RUSSELL et al., 1994, 1996), visandoa intervenção para reduzir a ingestão de álcool durante a gravidez. O questionário TWEAK consiste em uma escala de cinco itens desenvolvidos originalmente sobre o acrônimo abaixo e suas respectivas pontuações (Tabela 5):

Tabela 5. Questionário TWEAK.

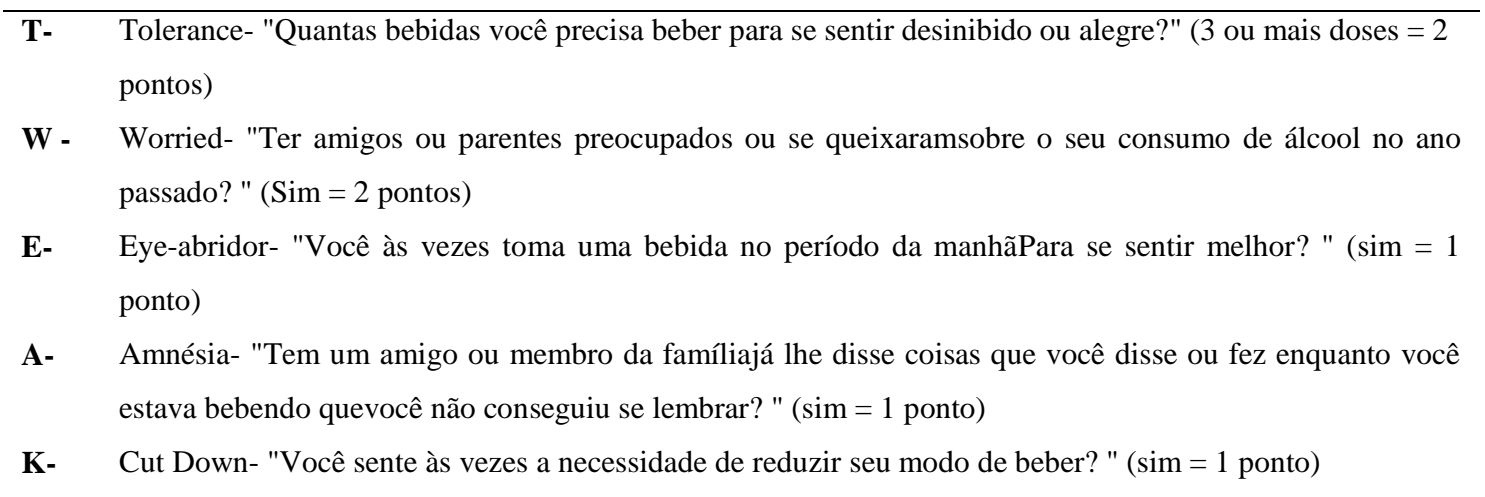

\section{Questionário CRAFFT}

SegundoKandemiret al (2015) é uma ferramenta de rastreio em forma de 6 perguntas utilizadas para identificar pacientes com alto risco de um diagnóstico de abuso ou dependência de substância. Cada resposta afirmativa equivele a um ponto, escore de 1 ponto indica necessidade de prevenção, escore $\geq 2$ indica consumo abusivo.

Tabela 6. Questionário de CRAFFT. 


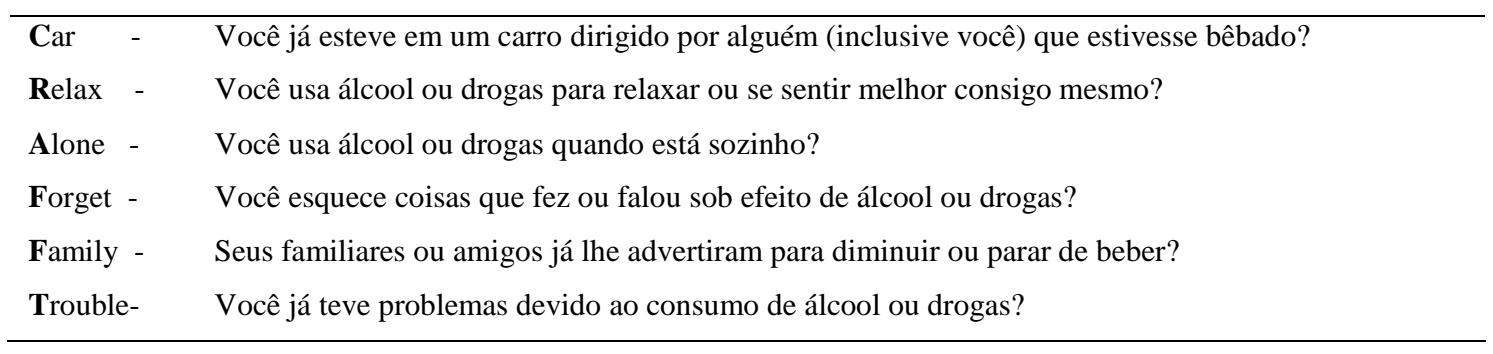

\section{Discussão}

Como avaliar o consumo de álcool pelo paciente odontológico? É difícil essa avaliação, seja pela omissão ou negação da informação, além de não ser uma pergunta rotineira do cirurgião-dentista.Geralmente, os pacientes não reconhecem seu vício e, naturalmente, nenhum deles considera seu consumo como excessivo, classificando-o como "bebo socialmente". Porém, é de extrema importância quantificar a frequência e a quantidade de álcool consumido pelo paciente. A LEI SECA nº 12.760/2012 que alterou o Código de Trânsito Brasileiro, estabeleceu que a concentração de 6 decigramas de álcool (2 latas de cerveja, 2 copos de vinho ou 1/2 copo de destilado)por litro de sangue comprova que o condutor está sob estado de embriaguez alcoólica e é classificado como crime.

Segundo pesquisa no Hospital de Clínicas de São Paulo, cerca de 15\% da população brasileira sofre de alcoolismo.A definição empírica aceita pela maioria dos médicos é que devem ser considerados consumidores moderados de álcool os homens que bebem o equivalente a 25 gramas por dia. Por diferenças na capacidade de metabolizar o álcool, nas mulheres esse limite equivaleria a 12,5 gramas, metade da dose estabelecida para os homens. Duas latinhas de cerveja, dois copos de vinho ou duas doses de bebida destilada, equivalem mais ou menos às 28 gramas de álcool estabelecidas como limite para os homens(SKLOVSKY et al 1989; KRUSE 1984).

Pacientes não considerados alcoólatras, que consomem 25 gramas de álcool diariamente, apresentam alterações nos mecanismos de coagulação do sangue, aumentando o tempo de coagulação.SegundoRimmet al (1999), consumir uma dose experimental de álcool durante 1 a 9 semanas demonstrou mudanças nas concentrações de fatores da coagulação como fibrinogênio, fator VII, fator de von Willebrand, tipo de atividade do ativador do plasminogênio tecidual e tipo de tecido antígeno ativador de plasminogênio. Com o sangue menos coagulável, haveria maior dificuldade para a formação de trombos nas artérias coronárias. 
COPYRIGHT @ 2018 INTERNATIONAL JOURNAL OF SCIENCE DENTISTRY | AVAILABLE ONLINE http://www.periodicos.uff.br/index

A ingestão de quantidades maiores de álcool, no entanto, reverte essa relação, favorecendo a coagulação mais rápida e a trombogênese. Esse fato conduz o paciente à lesões hepáticas. Considerando o fígado o órgão principal no processo da coagulação, doenças hepáticas podem levar a anormalidades na hemostasia por diversos mecanismos: defeito plaquetário, diminuição na produção de fatores da coagulação e de inibidores, síntese de fatores anormais da coagulação, hiperfibrinólise (SOUSA E SILVA, 2010), prejudicando o paciente frente a algum tratamento odontológicocirúrgico, devido à alteração em seu tempo de sangramento e a possibilidade de sofrer hemorragias pós-operatórias. Além disso, o álcool interfere na cicatrização de feridas, interações medicamentosas e atua no estado psíquico do paciente, prejudicando, muitas vezes, a colaboração deste frente às suas responsabilidades com o tratamento.

Sendo assim, é de suma importância o conhecimento do cirurgião dentista à respeito dos possíveis instrumentos, aplicáveis na clínica, para avaliação do consumo de álcool.

O questionário AUDIT é o que consegue diagnosticar todos os níveis de consumo de álcool apresentando conjuntos de itens pertencentes à três dimensões (quantidade/frequência, dependência, problemas/consequências adversas) que podem ser analisadas separadamente, permitindo uma caracterização mais especifica do paciente.A desvantagem é que o questionário AUDIT é o mais longo de todos e exige mais tempo do profisional de saúde para a entrevista.

O MAST, tem a vantagem de apresentar um número menor de itens, ser mais fácil de responder, sendo mais específico que o CAGE, T-ACE, TWEAK e CRAFFT.

O questionário CAGE é curto e de fácil itilização, porém apresenta como desvantagem o fato de requerer uma interpretação dos resultados.

O T-ACE se usado isoladamente pode não detectar problemas de abuso de álcool Sarkar et al (2010).

No questionário T-WEAK um escore de 2 (de 7 totais) já indica uso abusivo de alcool, é um questionário curto e simples, assim como o questionátio CRAFFT, que indica necessidade de prevenção com a obtenção de apenas 1 ponto e classifica como uso abusivo o paciente que alcança 2 pontos do total de 6 pontos.

Na prática clínica e com o intuito de caracterizar o paciente abusivo de álcool, o cirurgião-dentista pode, baseando-se no objetivo do diagnóstico e no tempo disponível 
COPYRIGHT @ 2018 INTERNATIONAL JOURNAL OF SCIENCE DENTISTRY | AVAILABLE ONLINE

http://www.periodicos.uff.br/index

para questionamento, optar pela utilização de um dos questionários apresentados. No entanto, cabe ressaltar que o questionário AUDIT vem sendo utilizado em inúmeras pesquisas e, apesar de demandar maior tempo de anamnese, apresenta detalhamento e padrões diferenciados para a caracterização do paciente.

\section{Conclusão}

São inúmeras as dificuldades acerca da determinação exata do consumo etílicodo paciente odontológico.No entanto, é essencial identificar a quantidade e frequência aceitáveis de consumo de álcool, que não colocariam em risco o resultado do tratamento e a saúde geral do paciente.

Os questionários podem quantificar a dose a partir da frequiência do consumo, medidas de concentração-freqüência, freqüências graduadas, memória recente do consumo e mensuração diária. Tudo isso produz dados de natureza absolutamente específica relacionada à dependência psicossomática, mas a determinação das condições gerais de saúde do paciente só podem ser determinada através de exames complementares.

\section{ReferênciasBibliográficas}

BABOR, T.F.; DE LA FUENTE, J.R.; et al . The Alcohol Use Disorders Identification Test. Guidelines for Use in Primary Health Care . Geneva, Switzerland: World Health Organization, 1992

CROTON, G. Screening for and assessment of co-occurring substance use and mental health disorders by Alcohol \& Other Drug and Mental Health Services., Victorian Dual Diagnosis Initiative Advisory Group, 2007.

DAWE S, R.V.W.; MATTICK, R.; et al. Efficacy of moderation-oriented cue exposure for problem drinkers: a randomized controlled trial. $\mathrm{J}$ Consult Clin Psychol. 2002;70(4):1045-50.

KANDEMIR, H.; AYDEMIR, O.; et al. Validity and reliability of the Turkish version of CRAFFT substance abuse screening Test among adolescentes, Neuropsychiatr Dis Treat 2015;11:1505-1509.

KNIBBE, R.A.; DERICKX, M.; et al.A comparison of the Alcohol Use Disorder Identification Test (AUDIT) in general population surveys in nine European countries.Alcohol Alcohol Suppl. 2006;41(1):i19-25.

KRUSE, J. Alcohol use during pregnancy. AmFam Physician, v. 29, p 199-203, 1984.

LEI SECA $\mathrm{n}^{\circ}$ 12.760/2012, http://www.planalto.gov.br/ccivil_03/_Ato20112014/2012/Lei/L12760.htm

LIMA, C.T.; FREIRE, A.C.; et al.Concurrent and construct validity of the audit in an urban brazilian sample. Alcohol Alcohol. 2005;40(6):584-589. 
COPYRIGHT @ 2018 INTERNATIONAL JOURNAL OF SCIENCE DENTISTRY | AVAILABLE ONLINE

http://www.periodicos.uff.br/index

MASUR, J.; DE SOUZA, M.L.; et al.Lack of effect of intravenous hypertonic glucose on the intensity of alcohol intoxication induced experimentally and observed in patients of an emergency room. Pharmacology. 1983;26(1):54-60.

MAYFIELD, D.; MCLEOD, G.; et al.The CAGE questionnaire: validation of a new alcoholism screening instrument.Am J Psych. 1974;131:1121-23.

MÉNDEZ, E.B. Uma versão brasileira do AUDIT - Alcohol Use DisordersIdentification Test. Dissertação de Mestrado não publicada, Universidade Federal de Pelotas, RS, 1999.

POKORNY, A.D.; MILLER, B.A.; et al.The Brief MAST: A shortened version of the Michigan Alcoholism Screening Test. Am J Psychiatry. 1972;129(3): 342-345.

PILLON, S.C.; LUIS, M.A.; et al. Nurses' training on dealing with alcohol and drug abuse: a question of necessity. Rev HospClinFac Med Sao Paulo. 2003;58(2):11924.

RIMM, E.B.; WILLIAMS, P.; et al. Moderate alcohol consumption and lower risk of coronary heart disease: metaanalysis of effects on lipids and hemostatic factors. BMJ. 1999;319:1523-8.

ROCHA, P.R.; DAVID, H.M.S.L.Questionários sobre o uso de álcool e drogas entre trabalhadores: revisão da literatura, SMAD.2011;7(2):107-116.

ROSÁRIO, A.M.M. Avaliação de instrumentos que investigam abuso de álcool e outras drogas em adolescentes: revisão de literatura. Dissertação de Mestrado, Faculdade de Medicina da Universidade de São Paulo, Programa de medicina Preventiva, 2011.

RUSSELL, M.; MARTIER, S. S.; et al. Screening for pregnancy riskdrinking. AlcoholClinExp Res. 1994;18:1156-1161.

RUSSELL, M.; MARTIER, S. S.; et al. Detecting risk drinking during pregnancy: A com parison of four screening questionnaires.Am J Public Health. 1996;86:14351439.

SARKAR, M.; EINARSON, T.; et al. Comparing the effectiveness of TWEAK and TACE in determining problem drinkers in pregnancy. Alcohol Alcohol. 2010;45(4):356-60.

SAUNDERS, J.B.; AASLAND, O.G.; et al. Development of the Alcohol Use Disorders Identification Test (AUDIT): WHO Collaborative Project on Early Detection of Persons with Harmful Alcohol Consumption--II. Addiction. 1993;88(6):791-804.

SKLOVSKY, E.; SILVEIRA, L.F.G.; et al. Adição de Drogas na Gravidez. Rev HCPA 1989;9(3):195-201.

SOKOL, R.J.; MARTIER, S.S.; et al.The T-ACE questions: practical prenatal detection of risk-drinking. Am J ObstetGynecol. 1989;160(4):863-8.

SOUSA E SILVA, I.S. Cirrose Hepática. RBM.2010;67(4):111-120. 